\title{
Simulating creep deformation in generalized visco-elastic medium with the space-time finite element method
}

\author{
Honggang Jia ${ }^{a}, \quad$ Yufeng Nie ${ }^{b}$ \\ School of Science, Northwestern Polytechnical University, Xian 710129, China \\ a z770428@126.com, byfnie@nwpu.edu.cn
}

Keywords: space-time finite element; generalised viscoelastic materials;creep

\begin{abstract}
The creep deformation of viscoelastic sample are simulated by apace-time finite element method/extended finite element method (XFEM). Two rheological models of 7-parameters generalized Kelvin-Voigt model and Burgers model are used to simulate the creep of sample under uniaxial or biaxial compressive, numerical examples shows that the accuracy of 7-parameters generalised Kelvin-Voigt model is better than that of Burgers model. The predicted creep results compared with that of literature reported is well.
\end{abstract}

\section{Introduction}

Space-time finite element method has become a widely used numerical tool in engineering and computation, finite element method for dynamic problems are based on semidiscretizations: finite elements are used in space to reduce to a system of ordinary differential equations in time, It is generally believed that finite elements represent a superior methodology to finite differences. Hence, the method of space-time finite element[1-5] is proposed.

For creep or stress analysis in generalized viscoelastic material, the numerical methods[6-9] are often used for its complex time-dependent properties. However, creep analysis in generalized viscoelastic material with space-time finite element method is few and most numerical methods, such as springs-dashpots viscoelastic models assembled in parallel or in serials, howerver, the numerical model using assembled in parallel or in serials, i.e., generalised viscoelastic model is very few. A recent paper about this area is reported in[9], in this study, it was shown that the generalised creep model is better than finite difference method are in terms of memory and computational time.

In this paper, space-time finite element method is applied to linear viscoelastic creep analysis in generlised viscoelastic material. Numerical examples validated its accuracy and efficiency.

In Section 2, the viscoelastic dynamic equations and linear viscoelastic constitutive equations are presented. In Section 3, the space-time finite element method is briefly stated, the generalized maxwell model and generalized Kelvin-Voigt model are given, Several numerical examples are given in Section 4, the conclusion are made in Section 5.

\section{Linear viscoelastic equations}

Consider an elastic body with bounded region, $\Omega \subset \mathfrak{R}^{3}$. Let $\Gamma$ represents the boundary of $\Omega,{ }_{g}, \Gamma_{h}$ the nonoverlapping subregions of $\Gamma_{\text {and }} \Gamma_{g} \cap \Gamma_{h}=\phi$. The vector of displacement is denoted as $u(x, t)$, here, $x \in \bar{\Omega}$ and $t \in[0, T]$.The stress-strain relationship can be written as

$$
\sigma_{i j}=c_{i j k l} u_{k, l}
$$

here, $1 \leq i, j, k, l \leq 3, u_{k, l}=\partial u_{k} / \partial x_{l}, c_{i j k l}$ are elastic coefficients.

The equations of the initial/boundary-value problem of elastodynamics are represented as[1] 


$$
\begin{aligned}
& \rho \ddot{\boldsymbol{u}}=\nabla \cdot \boldsymbol{\sigma}(\nabla \boldsymbol{u})+\boldsymbol{f} \text { on } \Omega \times] 0, T[ \\
& \left.\boldsymbol{u}=\overline{\boldsymbol{u}} \quad \text { on } \Gamma_{g} \times\right] 0, T[ \\
& \left.\boldsymbol{n} \cdot \boldsymbol{\sigma}=\overline{\boldsymbol{t}} \quad \text { on } \Gamma_{h} \times\right] 0, T[ \\
& \boldsymbol{u}(\boldsymbol{x}, 0)=\boldsymbol{u}_{0}(\boldsymbol{x}), \boldsymbol{x} \in \Omega \\
& \dot{\boldsymbol{u}}(\boldsymbol{x}, 0)=\boldsymbol{v}_{0}(\boldsymbol{x}), \quad \boldsymbol{x} \in \Omega
\end{aligned}
$$

here, $\rho$ is the density, a superposed dot represents differentiation with respect to $t, f$ denotes body force, $\overline{\boldsymbol{u}}, \overline{\boldsymbol{t}}$ are the prescribed displacement and traction on the boundary, respectively,

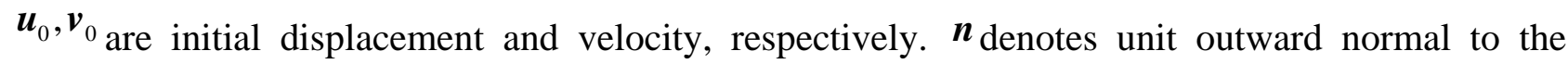
boundary. Fig. 1(cited from [6]) depicts four rheological models.

For arbitrary linear viscoelastic material, the rheological model can be composed of spring and dashpot elements assembled in series or parallel,
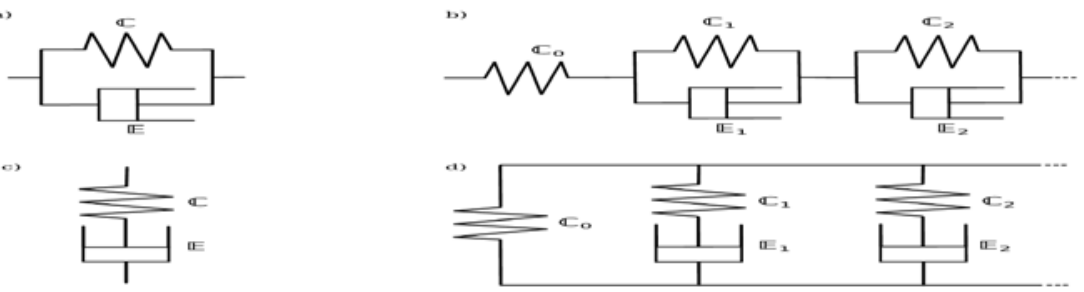

Fig. 1. Rheological models for linear visco-elasticity: a) Kelvin-Voigt model, b) generalised

Kelvin-Voigt model. c) Maxwell model , d) generalised Maxwell model.

For generalised Kelvin-Voigt model, its constitutive equation reads as

$$
\varepsilon(t)=\sigma_{0}:\left[C_{0}^{-1}+\sum_{k=1}^{n} C_{k}^{-1}\left(1-e^{-t / \tau_{k}}\right)\right]
$$

here, $\sigma_{0}$ is a constant force applied initially, ${ }^{C_{k}}$ denotes elastic stiffness tensor of $k$ th spring, ${ }^{\tau_{k}}$ denotes delay time of $k$ th dashpot.

For Burgers model, its constitutive equation reads as

$$
\varepsilon(t)=\sigma_{0}:\left[\frac{1}{E_{2}}+\frac{t}{\eta_{2}}+\frac{1}{E_{1}}\left(1-e^{-\frac{E_{1}}{\eta_{1}} t}\right)\right]
$$

\section{Space-time finite element method}

The space-time finite element method, also is a discontinuous Galerkin methods, have been used to solve time-dependent problems, such as dynamic problems. The method bases on using usual finite element discretizations in space and time simultaneously having basis functions which are continuous in space and discontinuous at the discrete time slab. In space-time finite element, the displacement, velocity, and acceleration are function of space coordinates and time coordinate, the basis function is multiplying space basis function by time basis function. The space-time displacement at arbitrary space and time can read as

$$
\mathbf{u}^{h}(x, y, z, t)=\sum_{i=1}^{k} N_{i}(x, y, z, t) a_{i}
$$


here, $\boldsymbol{N}_{i}$ are the usual finite element shape functions, $\boldsymbol{a}_{i}$ is nodes displacement freedom vector.

\section{Numerical examples}

\section{Example 1:biaixal creep}

Here, in order to use Burger's model, we consider a 2D generalized viscoelastic square sample whose size is $2 \mathrm{~m} \times 2 \mathrm{~m}$, the geometry and boundary conditions as are shown in Fig. 2. The material properties are cited from those of example in [6](p.80). Fig. 3 shows the simulated biaxial creep of the sample in the $\mathrm{x}$ and $\mathrm{y}$ directions. From it, we can find that the creep in $\mathrm{x}$ and $\mathrm{y}$ directions is same, which is due to the sample is symmetrical. The creep inreases with time under constant stress in $\mathrm{x}$ and y directions.

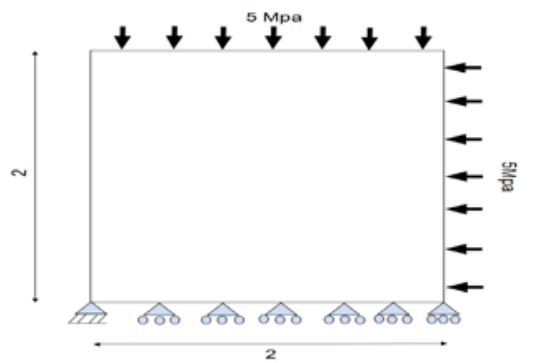

Fig. 2. 2D biaxial creep sample.

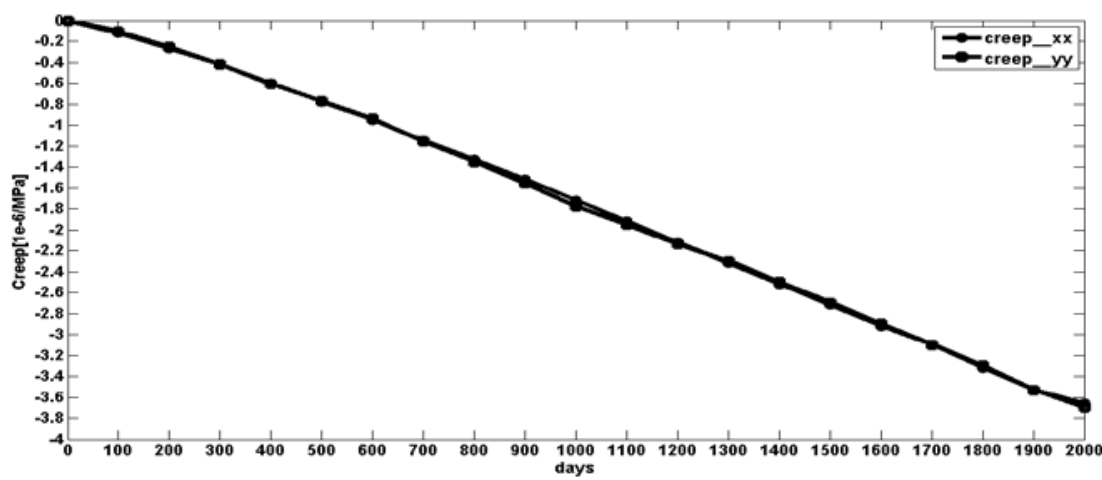

Fig. 3. Simulated Burger’s model biaixal creep.

\section{Example 2:uniaxial creep}

In this section, we consider a homogeneous rectangular plate of size $1 \mathrm{~m} \times 1 \mathrm{~m}$, a constant load of $1 \mathrm{~Pa}$ is applied on the plate, the geometry and boundary are illustrated in Fig. 4. Here, we adopt the generalized 4-parameters Kelvin-Voigt model.The Young's elastic moduli of the two 4-parameters Kelvin-Voigt elements of the generalized viscoelastic model is 4GPa, 4.5GPa for Kelvin-Voigt element 1 and Kelvin-Voigt element 2, respectively. The Characteristic time of the two Kelvin-Voigt elements is 10days, 300days for Kelvin-Voigt element 1 and Kelvin-Voigt element 2, respectively. The simulate creep function are reported in Fig. 5. From it, we can see that the creep function value increases with time, and the simulated numerical results agrees well with the experiment.

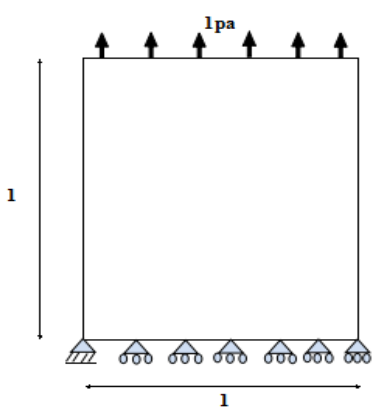

Fig. 4. The geometry and boundary of plate. 


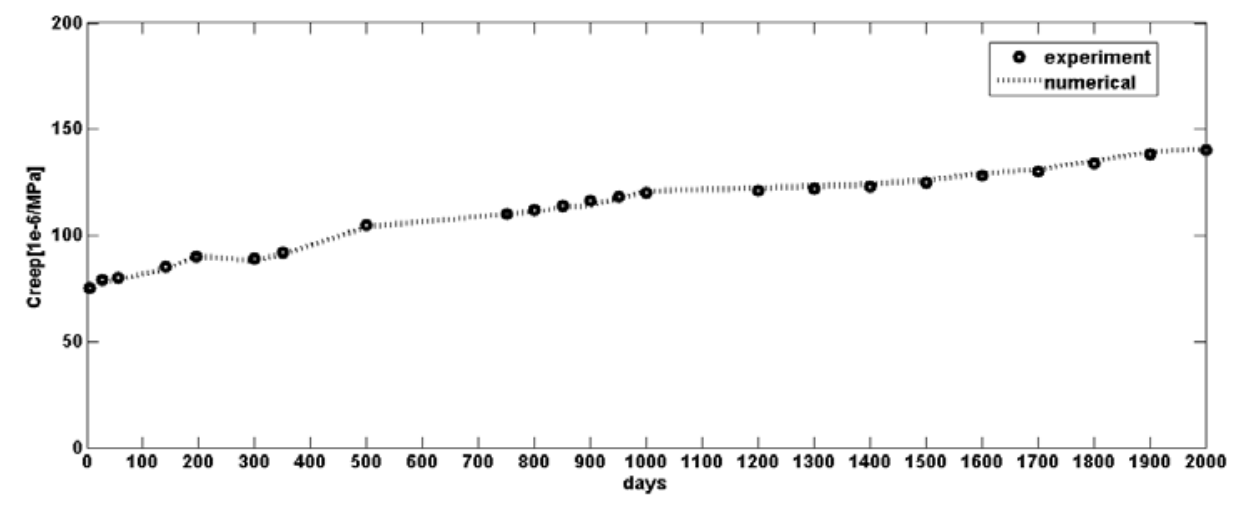

Fig. 5. Comparison of experiment data are cited from[10] with the numerical results.

\section{Summary}

In this contribution, we simulated uniaxial and biaxial creep behavior of generalized viscoelastic sample using space-time finite element method, from the simulations, we can make these conclusions as follows:

Under constant uniaxial load or biaxial load, sample creep increases with time.

The space-time finite element method can simulate creep behavior in arbitrary viscoelastic or generalized viscoelastic material.

\section{Acknowledgements}

This work was financially supported by the National Natural Science Foundation of China(11471262).

\section{References}

[1] J.R. Thomas Hughes and M. Gregory Hulbert:Comput.Method.Appl.M Vol. 66 (1988), p. 339-363

[2]A.Idesman,R.Niekamp and E.Stein:Comput.Meth.Appl.Mech.Eng Vol.190(2000),

p.1049-1063

[3] A.Idesman, R.Niekamp and E. Stein: Comput.Mech Vol.27(2001), p.49-60

[4] X.D. Li and N.E.Wiberg:Comput.Meth.Appl.Mech.Eng Vol.156(1998), p.211-229

[5]J.Chessa and T.Belytschko:Int. J. Numer. Meth. Engng Vol.61(2004), p.2595-2614

[6] A. B.Giorla: Modeling of Alkali-Silica Reaction under Multi-Axial load. Ph.D.thesis, Ecole Polytechnique Fédérale de Lausanne.(2013)

[7] A. B.Giorla, K. L.Scrivener and C. F.Dunant:Int. J.Numer.Meth.Eng Vol.97 (2014), p.454-472.

[8] C. F. Dunant: Experimental and Modelling Study of the Alkali-Silica-Reaction in Concrete. Ph.D.thesis, Ecole Polytechnique Fédérale de Lausanne. (2009)

[9]A. B.Giorla, K. L.Scrivener and C. F. Dunant: Int.J.Numer.Meth.Eng Vol. 97(2014), p.454-472

[10] A. Bengougam: Déformations différées et effets d’échelle des bétons de barrage, Ph.D.thesis, Ecole Polytechnique Fédérale de Lausanne. (2003) 